\title{
Comparative study of corticosteroid monotherapy, and TNF inhibitors with or without corticosteroid in patients with refractory entero-Behcet's disease
}

Ippei Miyagawa, Kazuhisa Nakano, Shigeru Iwata, Shingo Nakayamada, Kazuyoshi Saito, Kentaro Hanami, Shunsuke Fukuyo, Satoshi Kubo, Akio Kawabe, Yusuke Miyazaki and Yoshiya Tanaka (D)

\begin{abstract}
Background: Tumor necrosis factor (TNF) inhibitors (TNF-i) are effective in the treatment of entero-Behcet's disease (BD). However, there is no objective tool for assessment of disease activity in entero-BD; therefore, it is not easy to evaluate treatment effectiveness in the clinical setting. In addition, because corticosteroid (CS) is considered for standard therapy, the effectiveness of TNF-i without CS has not been well examined. In this retrospective study, the effectiveness of CS without TNF-i and the effectiveness of TNF-i with or without CS therapy were investigated and compared.
\end{abstract}

Methods: This study included 71 patients with entero-BD who were followed up for 1 year (CS without TNF-i group: $n=22$; TNF-i group: $n=49$ [with CS: $n=20$, without CS: $n=29$ ]). All patients had active ulcerative lesions. The primary endpoint was the ulcer cure rate evaluated by lower gastrointestinal endoscopy. Secondary endpoints were ulcer improvement rate, disease activity improvement based on the quantitative disease activity index for intestinal Behcet's disease (DAIBD), and CS-sparing effect.

Results: Ulcer cure rates were $13.6 \%$ in the CS without TNF-i group, $60.0 \%$ in the TNF-i with CS group, and $44.8 \%$ in the TNF-i without CS group. Ulcer improvement rates were $27.2 \%$ in the CS without TNF-i group, $60.0 \%$ in the TNF-i with CS group, and $51.7 \%$ in the TNF-i without CS group. The multivariate analysis revealed that TNF-i was an independent predictive factor for cure of the ulcerative lesions. The DAIBD and concomitant CS dose were significantly decreased in both the CS without TNF-i group (DAIBD $85.2 \rightarrow 40.5$, CS $32.3 \rightarrow 18.7 \mathrm{mg} /$ day) and the TNF-i group (DAIBD 64.7 $\rightarrow$ 21.1. CS $18.7 \rightarrow 3.88 \mathrm{mg} /$ day). The ulcer cure and improvement rates were significantly higher in the TNF-i group. In addition, the proportion of concomitant CS dose less than $7.5 \mathrm{mg}$ was significantly higher in the TNF-i group (CS without TNF-i group $18.2 \%$ vs. TNF-i group $85 \%, P<0.01$ ). There were no statistically significant differences between the TNF-i with CS group and the TNF-i without CS group in any of the endpoints.

Conclusions: This study demonstrated that compared to CS alone, TNF-i improve disease activity and possess a higher ulcer healing effect and CS tapering effect with or without concomitant CS.

Keywords: Behcet's disease, Entero-Behcet's disease, TNF inhibitor, Corticosteroid, Disease activity index

\footnotetext{
* Correspondence: tanaka@med.uoeh-u.ac.jp

The First Department of Internal Medicine, School of Medicine, University of

Occupational and Environmental Health, 1-1 Iseigaoka, Yahata-nishi,

Kitakyushu 807-8555, Japan
}

(c) The Author(s). 2019 Open Access This article is distributed under the terms of the Creative Commons Attribution 4.0 International License (http://creativecommons.org/licenses/by/4.0/), which permits unrestricted use, distribution, and reproduction in any medium, provided you give appropriate credit to the original author(s) and the source, provide a link to the Creative Commons license, and indicate if changes were made. The Creative Commons Public Domain Dedication waiver (http://creativecommons.org/publicdomain/zero/1.0/) applies to the data made available in this article, unless otherwise stated. 


\section{Background}

Behcet's disease (BD) is a relapsing, systemic inflammatory disease that affects the skin, mucosa, eyes, genitourinary organs, joints, blood vessels, central nervous system, and gastrointestinal system [1]. Entero-Behcet's disease (enteroBD) is often associated with bleeding and perforation, which is refractory to therapy. Many patients with entero$\mathrm{BD}$ are resistant to conventional treatment with corticosteroid (CS) and immunosuppressive drugs, and some require surgery. We and other groups have recently shown that TNF inhibitors (TNF-i) are effective in the treatment of entero-BD [2-8]. However, there is no objective tool for assessment of disease activity in entero-BD; therefore, it is not easy to evaluate disease activity and treatment efficacy in the clinical setting. In addition, because CS is considered for standard therapy in patients with severe symptoms and with deep ulcerative lesions [9, 10], the effectiveness of TNF-i without CS has not been well examined [11]. In the present study, we quantitatively investigated the effectiveness of 1-year treatment with TNF-i in 49 patients with entero-BD refractory to conventional therapy and compared the results to those achieved with CS without TNF-i, using the disease activity index for intestinal Behcet's disease (DAIBD) developed in 2011 [12, 13]. We also investigated effectiveness of TNF-i without CS and compared to that of TNF-i with CS.

\section{Patients and methods}

\section{Patients}

The present study included 71 patients diagnosed with entero-BD according to the revised diagnostic criteria proposed by the Behcet's Disease Research Committee of Japan (2003) and the criteria for diagnosis of BD recommended by the International Study Group [14, 15]. All 71 patients had active ulcerative lesions. Patients with diseases similar to $\mathrm{BD}$, such as intestinal tuberculosis, Crohn's disease, ulcerative colitis, infectious colitis, or simple ulcers, were excluded. Patients who were newly introduced the remission induction therapy mainly with CS but without TNF-i for active ulcerative lesions at our hospital were defined as CS without TNF-i group, regardless of past and recent treatment and dose of concomitant CS. Likewise, patients newly introduced the remission induction therapy with TNF-i for active ulcerative lesions at our hospital were defined as the TNF-i group, regardless of past and recent treatment and use and dose of concomitant CS. Choices regarding type of TNF-i and use and dose of CS were made at the physicians' discretion. Remission induction therapy was defined as the treatment for newly emerging active ulcerative lesions. This study formed part of a research project investigating BD supported by the Health Labour Science Research Grant for research on rare and intractable diseases. The Human Ethics Review Committee of our university reviewed and approved this study. All participants provided informed consent prior to inclusion in the study. Details that might disclose the identity of study subjects were omitted.

\section{Clinical measurement}

In the present study, we investigated the effectiveness of 1-year treatment with TNF-i and compared the results to those achieved with CS without TNF-i. We also investigated effectiveness of TNF-i without CS and compared to that of TNF-i with CS.

Remission induction therapy mainly with CS but without TNF-i (22 patients) and remission induction therapy with TNF-i (49 patients; 20 TNF-i with CS, 29 TNF-i without CS) were newly introduced at our hospital. Study patients were followed up for 1 year after the introduction of the treatments at our hospital and affiliated institutions, as shown in Fig. 1. The primary endpoint was the ulcer cure rate, as evaluated by lower gastrointestinal endoscopy 1 year after the introduction of remission induction therapy with either TNF-i or CS without TNF-i. "Cure" was defined as disappearance and scarring of ulcerative lesions. "Improvement" was defined as cure, scarring, and reduction of ulcerative lesions. In the case of patients who were unable to undergo lower gastrointestinal endoscopy owing to abdominal pain or for other reasons, the status was classified as "Exacerbation." In the TNF-i without CS group, in patients for whom concomitant CS was newly introduced, the status was classified as "Exacerbation." Likewise, in the CS without TNF-i group, in patients for whom concomitant CS was increased, the status was classified as "Exacerbation." The secondary endpoints were the ulcer improvement rate, ulcer exacerbation rate, and continuation rate of TNF-i. Regarding the continuation rate of the TNF-i without CS group, in patients for whom CS was newly introduced, the status was classified as "Discontinuation." We also analyzed disease activity improvement based on the DAIBD and the CS-sparing effect after 1 year of treatment.

The DAIBD (Disease Activity Index for intestinal Behcet's disease) is a quantitative disease activity index proposed by Cheon et al. in 2011 [14, 15]. Prior to the development of the DAIBD, there had been no useful and objective tool to assess the disease activity of entero-BD. The DAIBD (total score, 0-285) consists of eight items: (i) general well-being for 1 week, (ii) fever, (iii) extraintestinal manifestations, (iv) abdominal pain in 1 week, (v) abdominal mass, (vi) abdominal tenderness, (vii) intestinal complications, and (viii) number of liquid stools in 1 week.

\section{Statistical analysis}

Data are expressed as mean \pm standard deviation (SD). Differences between the groups and between baseline data 


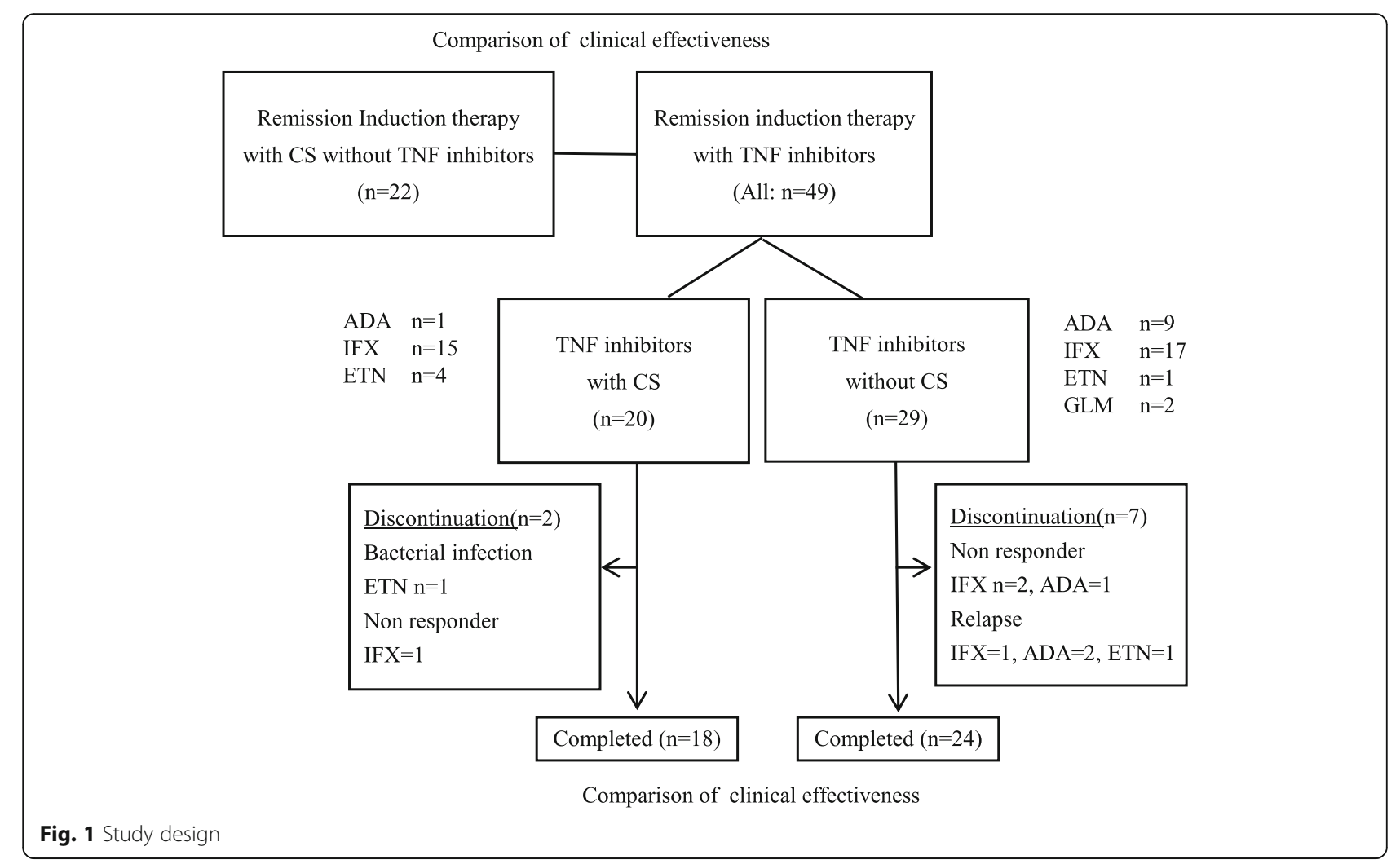

and data measured at 1 year were compared using Fisher's exact test, the Mann-Whitney $U$ test, and the Wilcoxon signed-rank test. The patients were classified into groups with cure of intestinal ulcerative lesions or not, or with achieving CS less than $7.5 \mathrm{mg} /$ day group or not. In addition, univariate and multivariate logistic regression analyses were performed on these groups. In the multivariate analysis, the proportion of cure of intestinal ulcerative lesions or achieving CS less than $7.5 \mathrm{mg} /$ day at year 1 were used as a dependent variable. All reported $P$ values were two-sided. The level of significance was set at $P<0.05$.

All analyses were conducted using JMP version 10.0 (SAS Institute Inc., Cary, NC, USA). For statistical analysis, data from TNF-i discontinued cases were complemented using the last observation carried forward method.

\section{Results}

\section{Patient background}

Table 1 shows the patient background at baseline. All patients in the present study were diagnosed with entero$\mathrm{BD}$ based on the existence of the typical ulcerative lesions on gastrointestinal endoscopy. In the group with CS without TNF-i (22 patients), the mean patient age was 34.1 years. The median dose of concomitant CS was $30 \mathrm{mg} /$ day (prednisolone equivalent). The mean DAIBD was 85.2. In the group with TNF-i and CS group (20 patients), the mean patient age was 42.2 years. The median dose of concomitant corticosteroid was $10 \mathrm{mg} /$ day (prednisolone equivalent). The mean DAIBD was 70.7. In contrast, in the group with TNF-i without CS (29 patients), the mean patient age was 40.7 years. Twenty-one patients had no history of TNF-i treatment (Bio naive). The mean DAIBD was 60.5. Although the concomitant CS dose and the DAIBD were statistically significantly higher in the group with CS without TNF-i than in the group with TNF-i, there were almost no statistically significant differences in baseline background factors between the TNF-i with CS group and the TNF-i without CS group.

\section{Treatment effectiveness as evaluated by lower gastrointestinal endoscopy}

The ulcer cure rate at 1 year was $13.6 \%$ ( 3 of 22 patients) in the group with CS without TNF-i. In the same group, the ulcer improvement rate was $27.3 \%$ (6 of 22 patients). In contrast, the ulcer cure rates at 1 year were $60.0 \%$ (12 of 20 patients) in the group with TNF-i and CS, and $44.8 \%$ (13 of 29 patients) in the group with TNF-i without CS. The ulcer improvement rates were $60.0 \%$ (12 of 20 patients) in the group with TNF-i and CS and 51.7\% (15 of 29 patients) in the group with TNF-i without CS. Although the ulcer cure and improvement rates were significantly lower in the group with CS without TNF-i than in the group with TNF-i, there were no significant differences in these outcomes between the TNF-i with CS group and the TNF-i without CS group (Fig. 2a, b). The 


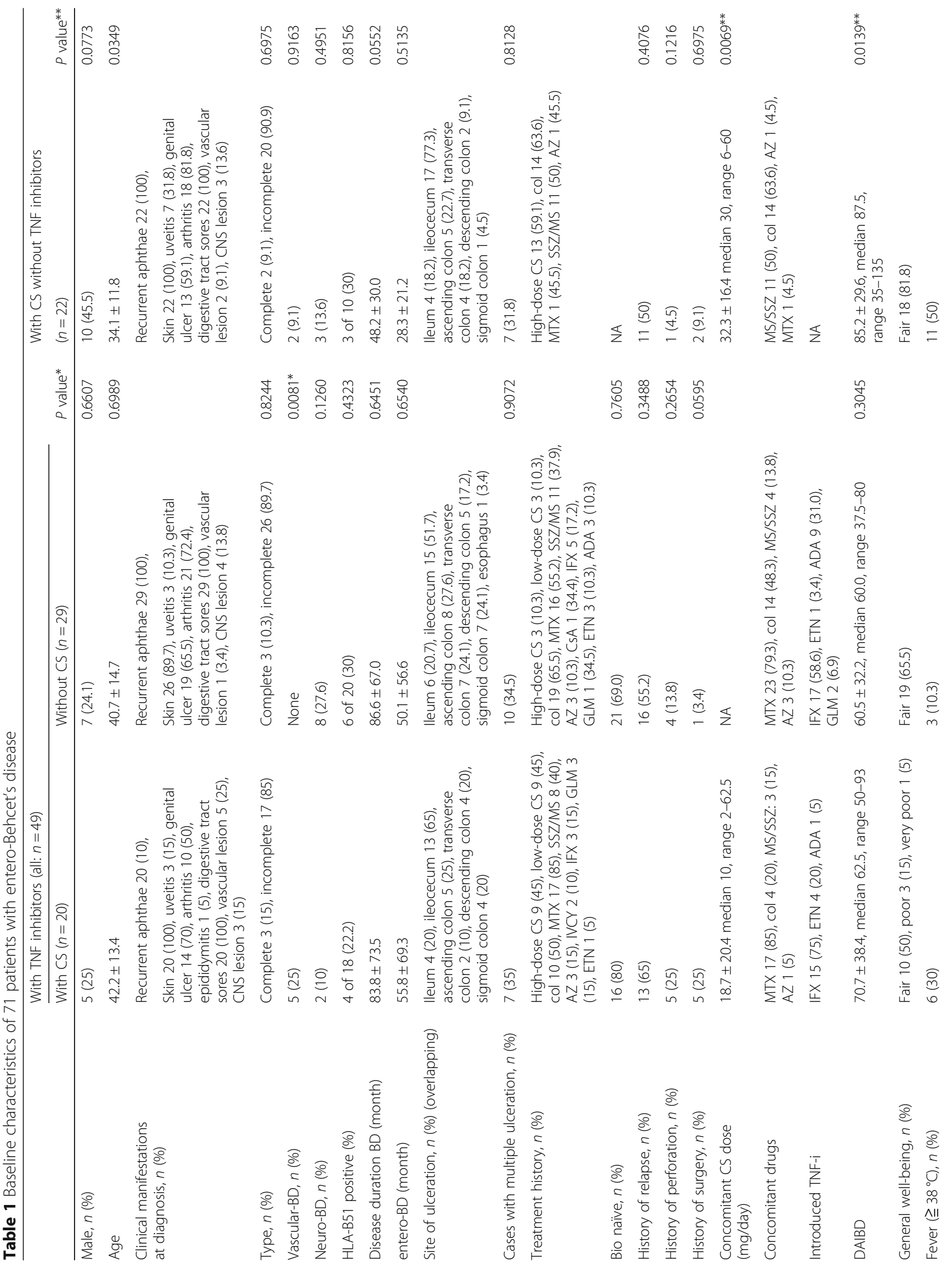




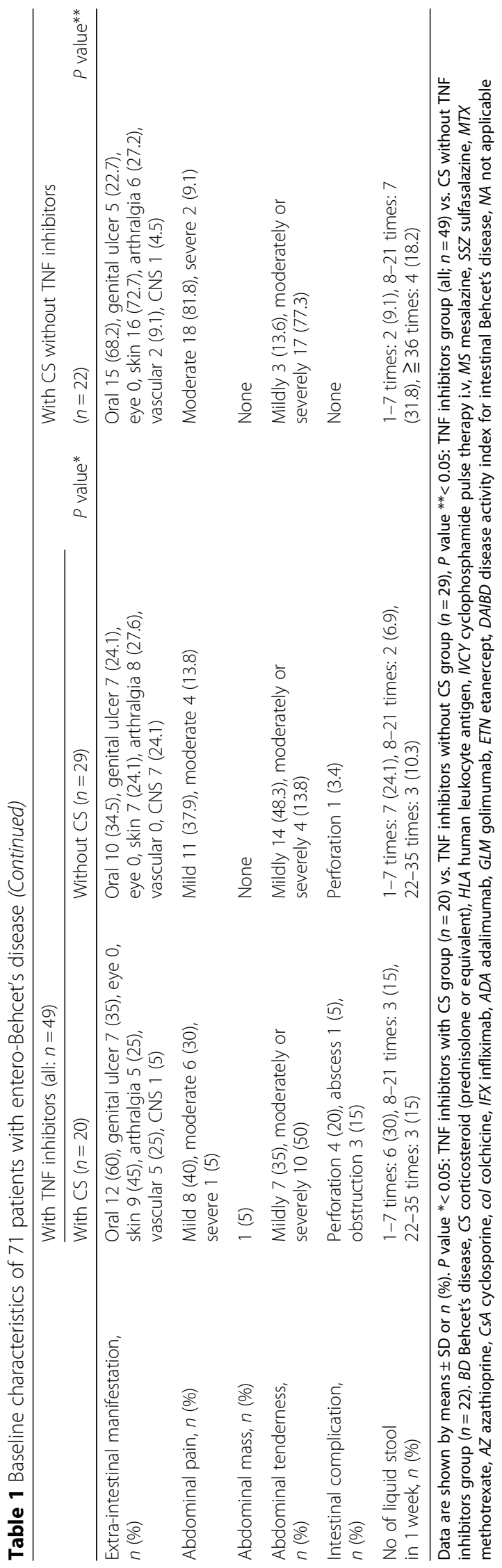


ulcer exacerbation rates were $20.0 \%$ ( 4 of 20 patients) in the group with TNF-i and CS and $29.8 \%$ (8 of 29 patients) in the group with TNF-i without CS $(P=0.3984)$ (Fig. 2c). During the observation period, three patients were newly started on CS in the group with TNF-i without CS, and the status in these patients was classified as "Exacerbation" and "Discontinuation." The data of these patients were complemented using the last observation carried forward method. In the present study, the cure of the intestinal ulcerative lesions was observed in 28 patients. When baseline characteristics were compared between patients with and without cure of the intestinal ulcerative lesions, as shown in Table 2, TNF-i was significantly more frequently used in patients with cure of the intestinal ulcerative lesions. In addition, univariate and multivariate logistic regression analyses were performed to identify the predictive factors for the cure of ulceration. The univariate logistic analysis identified colchicine, MTX, and TNF-i use and history of perforation with a $P$ value of $<0.1$. Subsequently, a multivariate logistic analysis was performed with these factors as dependent variables and identified the TNF-i use as an independent predictive factor $(P=0.0416$, odds ratio $6.17,95 \%$ confidence interval 1.01-37.8) (Table 3).

\section{Effectiveness of TNF inhibitors on improvement of} disease activity index for entero-Behcet's disease In the group with CS without TNF-i, the mean ( \pm SD) DAIBD was $85.2 \pm 29.6$ at baseline and $40.5 \pm 44.7$ at 1 year, showing a significant decrease. In the group with TNF-i, the mean $( \pm$ SD) DAIBD was also significantly decreased, from $64.7 \pm 34.9$ at baseline to $21.1 \pm 28.9$ at 1 year. In the group with TNF-i and CS, the mean $( \pm$ SD) DAIBD was $70.7 \pm 38.4$ at introduction and $20.8 \pm 29.3$ at 1 year, demonstrating a significant decrease. Likewise, the mean $( \pm$ SD) DAIBD was $60.5 \pm 32.2$ at introduction and $21.4 \pm 25.5$ at 1 year, showing a significant decrease in the group with TNF-i without CS (Fig. 3a). Comparison of the changes in DAIBD between the CS without TNF-i and TNF-i groups, and between the TNF-i with CS and TNF-i without CS groups, revealed no statistically significant differences (CS without TNF-i group $44.8 \pm 47.1$ vs. TNF-i $43.6 \pm 37.2$, TNF-i with CS group $50 \pm 38.3$ vs. TNF-i without CS group $39.1 \pm 36.4$ ) (Fig. 3b).

\section{Effectiveness of TNF inhibitor on reduction of concomitant CS dose}

In the group with CS without TNF-i, the mean $( \pm \mathrm{SD})$ dose of concomitant CS was $32.3 \pm 16.4 \mathrm{mg} /$ day at baseline and $20.6 \pm 18.3 \mathrm{mg} /$ day at 1 year, demonstrating a significant decrease. Likewise, the mean $( \pm S D)$ dose of concomitant CS in the group with TNF-i and CS was $18.7 \pm 20.9 \mathrm{mg} /$ day at baseline and $3.88 \pm 3.87 \mathrm{mg} /$ day at 1 year, showing a significant decrease (Fig. 4a). Comparison of the reduction of concomitant CS dose between the CS without TNF-i group and the TNF-i with CS group demonstrated no statistically significant difference (CS without TNF-i group $11.7 \pm 23.5 \mathrm{mg}$ vs. TNF-i with CS

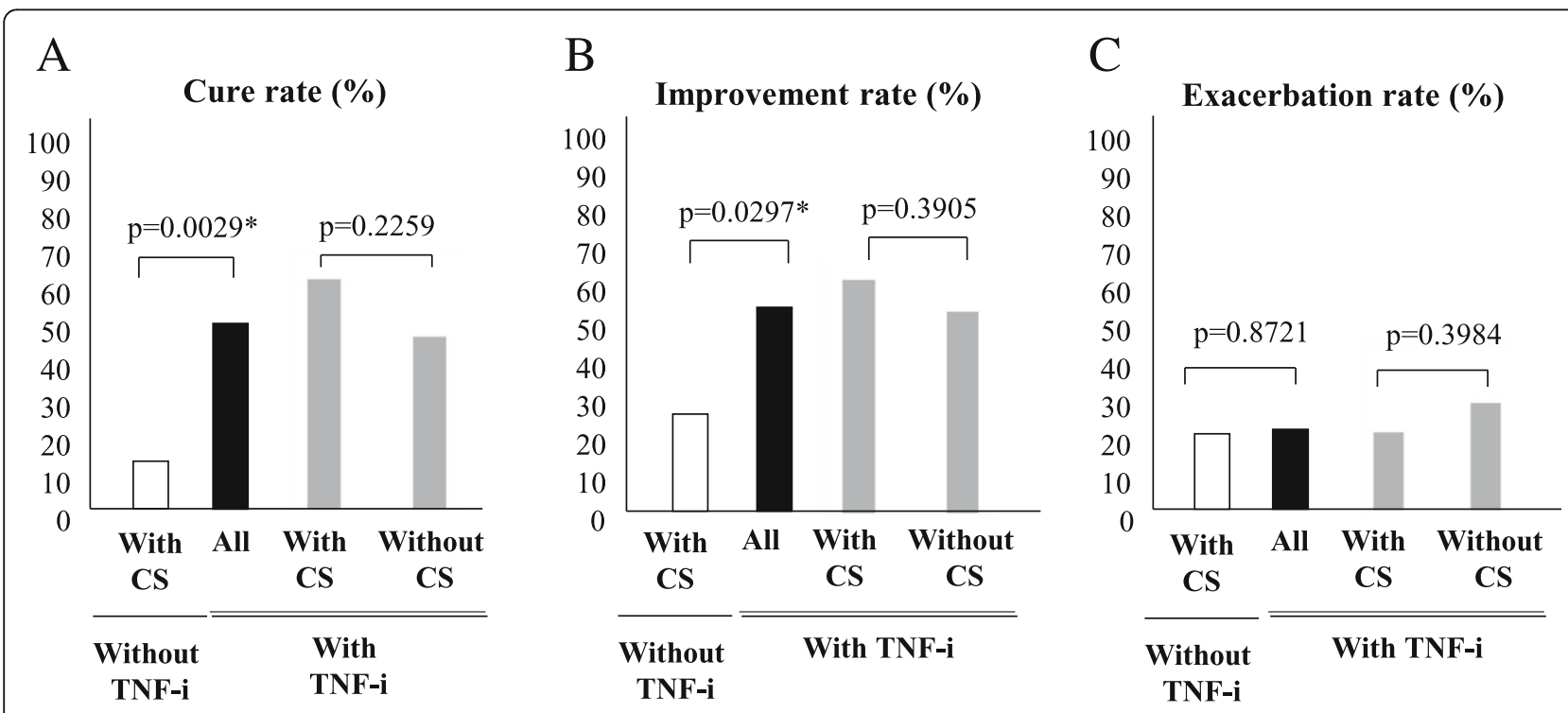

Fig. 2 Treatment effectiveness as evaluated by lower gastrointestinal endoscopy. a Ulcer cure rate, $\mathbf{b}$ improvement rate, and $\mathbf{c}$ exacerbation rate as evaluated by lower gastrointestinal endoscopy in the CS without TNF-i group $(n=22)$, TNF-i group (all: $n=49)$, TNF-i with CS group ( $n=20)$, and TNF-i without CS group $(n=29)\left({ }^{*} P<0.05\right.$, by Fisher's exact test) (last observation carried forward). Abbreviations: CS, corticosteroid; TNF-i, TNF inhibitors; DAIBD, disease activity index for intestinal Behcet's disease 
Table 2 Comparison of baseline characteristics between cure of the ulcerative lesions group and not

\begin{tabular}{|c|c|c|c|}
\hline Variables & $\begin{array}{l}\text { Cure of the } \\
\text { ulcerative lesions } \\
(n=28)\end{array}$ & Not $(n=43)$ & ${ }^{*} P$ value \\
\hline Male, $n(\%)$ & $10(35.7 \%)$ & $12(27.9 \%)$ & 0.6011 \\
\hline Age (years) & $40.2 \pm 12.3$ & $38.3 \pm 14.6$ & 0.2659 \\
\hline $\begin{array}{l}\text { Disease duration } \\
\text { (entero-BD, months) }\end{array}$ & $49.6 \pm 64.1$ & $41.9 \pm 45.6$ & 0.8923 \\
\hline $\begin{array}{l}\text { Multiple ulceration, } \\
n(\%)\end{array}$ & $7 / 28(25.0 \%)$ & $17 / 43(39.5 \%)$ & 0.3048 \\
\hline $\begin{array}{l}\text { History of relapse, } \\
n(\%)\end{array}$ & 16/28 (57.1\%) & $24 / 43(55.8 \%)$ & 1.0000 \\
\hline $\begin{array}{l}\text { History of perforation, } \\
n(\%)\end{array}$ & $7 / 28(25 \%)$ & $3 / 43(6.98 \%)$ & $0.0425^{*}$ \\
\hline $\begin{array}{l}\text { History of surgery, } \\
n(\%)\end{array}$ & $5 / 28(17.9 \%)$ & $3 / 43(6.98 \%)$ & 0.2495 \\
\hline $\begin{array}{l}\text { Concomitant CS use, } \\
n(\%)\end{array}$ & 15/28 (53.6\%) & $27 / 43(62.8 \%)$ & 0.4686 \\
\hline $\begin{array}{l}\text { Concomitant CS } \\
\text { dose (mg/day) }\end{array}$ & $14.5 \pm 20.5$ & $15.8 \pm 19.2$ & 0.5661 \\
\hline $\begin{array}{l}\text { Concomitant colchicine } \\
\text { use, } n(\%)\end{array}$ & $7 / 28(25 \%)$ & $23 / 43(53.5 \%)$ & $0.0265^{*}$ \\
\hline $\begin{array}{l}\text { Concomitant drug } \\
\text { use, } n(\%) \\
\text { (MTX, MS/SSZ, AZ) }\end{array}$ & 26/28 (92.9\%) & $34 / 43(79.1 \%)$ & 0.1812 \\
\hline $\begin{array}{l}\text { Concomitant MTX } \\
\text { use, } n(\%)\end{array}$ & $21 / 28(75 \%)$ & 20/43 (46.5\%) & $0.0265^{*}$ \\
\hline TNF-i use, $n(\%)$ & 25/28 (89.3\%) & $24 / 43(55.8 \%)$ & $0.0036^{*}$ \\
\hline DAIBD & $67.3 \pm 31.0$ & $73.5 \pm 36.7$ & 0.6332 \\
\hline
\end{tabular}

Plus-minus values are means \pm standard deviation. ${ }^{*} P<0.05$ by Fisher's exact test or Mann-Whitney $U$ test. BD Behcet's disease, CS corticosteroid, col colchicine, MS mesalazine, SSZ sulfasalazine, $M T X$ methotrexate, $A Z$ azathioprine, TNF- $i$ TNF inhibitors, DAIBD disease activity index for intestinal Behcet's disease group $14.9 \pm 21.0 \mathrm{mg}$ ) (Fig. 4b). In contrast, the proportions of patients receiving concomitant CS dose less than $7.5 \mathrm{mg}$ were significantly higher in the TNF-i with CS group than in the CS without TNF-i group (Fig. 4c). In the present study, achieving CS less than $7.5 \mathrm{mg} /$ day at year 1 was observed in 21 patients. When baseline characteristics were compared between patients with achieving CS less than $7.5 \mathrm{mg} /$ day or not, as shown in Table 4, MTX and TNF-i were significantly more frequently used and age was higher in patients with achieving CS less than 7.5 $\mathrm{mg} /$ day. In addition, univariate and multivariate logistic regression analyses were performed to identify the predictive factors achieving CS less than $7.5 \mathrm{mg} /$ day (Table 5). As the results, although TNF-i is close to being significant, no evident predictive factor was found. Furthermore, there were not any statistical differences in the baseline factors and clinical effectiveness between patients with and without MTX in the TNF-i group (Tables 6 and 7).

\section{Retention rate and adverse events of TNF inhibitor}

The retention rates up to month 12 were $90.0 \%$ (18 of 20 patients) in the group with TNF-i and CS and 75.9\% (22 of 29 patients) in the group with TNF-i without CS, demonstrating no significant differences $(P=0.1920)$. The status of 3 patients who newly started CS in the group with TNF-i without CS was classified as "Discontinuation." The reasons for discontinuation are summarized in Fig. 1. During the observation period, mild infection (cold) occurred in 10 patients, cystitis in two, viral enteritis in two, influenza in one, tonsillitis in one, bronchitis in one, sinusitis in one, paronychia in one, and urticaria in one. One patient in the group with

Table 3 Baseline predictive factors for cure of ulcerative lesions by multivariate analysis

\begin{tabular}{|c|c|c|c|c|c|c|}
\hline \multirow[t]{2}{*}{ Variables } & \multicolumn{3}{|c|}{ Univariate logistic regression } & \multicolumn{3}{|c|}{ Multiple logistic regression } \\
\hline & Wald & $P$ & OR $(95 \% \mathrm{Cl})$ & Wald & $P$ & OR $(95 \% \mathrm{Cl})$ \\
\hline Male & 0.4814 & 0.4878 & $0.70(0.25-1.93)$ & & & \\
\hline Age (years) & 0.3122 & 0.5763 & $0.99(0.96-1.01)$ & & & \\
\hline Disease duration (entero-BD) & 0.3496 & 0.5543 & $0.99(0.99-1.00)$ & & & \\
\hline Multiple ulceration & 1.5773 & 0.2091 & $1.96(0.69-5.61)$ & & & \\
\hline History of relapse & 0.0121 & 0.9121 & $0.95(0.36-2.48)$ & & & \\
\hline History of perforation & 4.0542 & 0.0345 & $0.23(0.05-0.96)$ & 2.4664 & 0.1163 & $0.28(0.06-1.38)$ \\
\hline History of surgery & 1.8818 & 0.1701 & $0.35(0.08-1.58)$ & & & \\
\hline Concomitant CS use & 0.5943 & 0.4407 & $1.46(0.56-3.84)$ & & & \\
\hline Concomitant CS dose (mg/day) & 0.07655 & 0.7820 & $1.00(0.98-1.03)$ & & & \\
\hline Concomitant colchicine use & 5.4007 & 0.0201 & $3.45(1.21-9.80)$ & 2.7596 & 0.0967 & $2.74(0.73-8.99)$ \\
\hline Concomitant drugs use (MTX, MS/SSZ, AZ) & 3.4411 & 0.1007 & $3.44(0.68-17.3)$ & & & \\
\hline Concomitant MTX use & 5.4008 & 0.0201 & $3.45(1.21-9.80)$ & 0.2392 & 0.6247 & $1.48(0.30-7.23)$ \\
\hline TNF-i use & 7.6116 & 0.0017 & $6.60(1.73-25.2))$ & 3.8795 & 0.0416 & $6.17(1.01-37.8)$ \\
\hline DAIBD & 0.5470 & 0.4595 & $1.01(0.99-1.02)$ & & & \\
\hline
\end{tabular}

Model $X^{2}$ test $P=0.0040$. BD Behcet's disease, CS corticosteroid, col colchicine, MS mesalazine, SSZ sulfasalazine, MTX methotrexate, $A Z$ azathioprine, TNF- $i$ TNF inhibitors, DAIBD disease activity index for intestinal Behcet's disease 


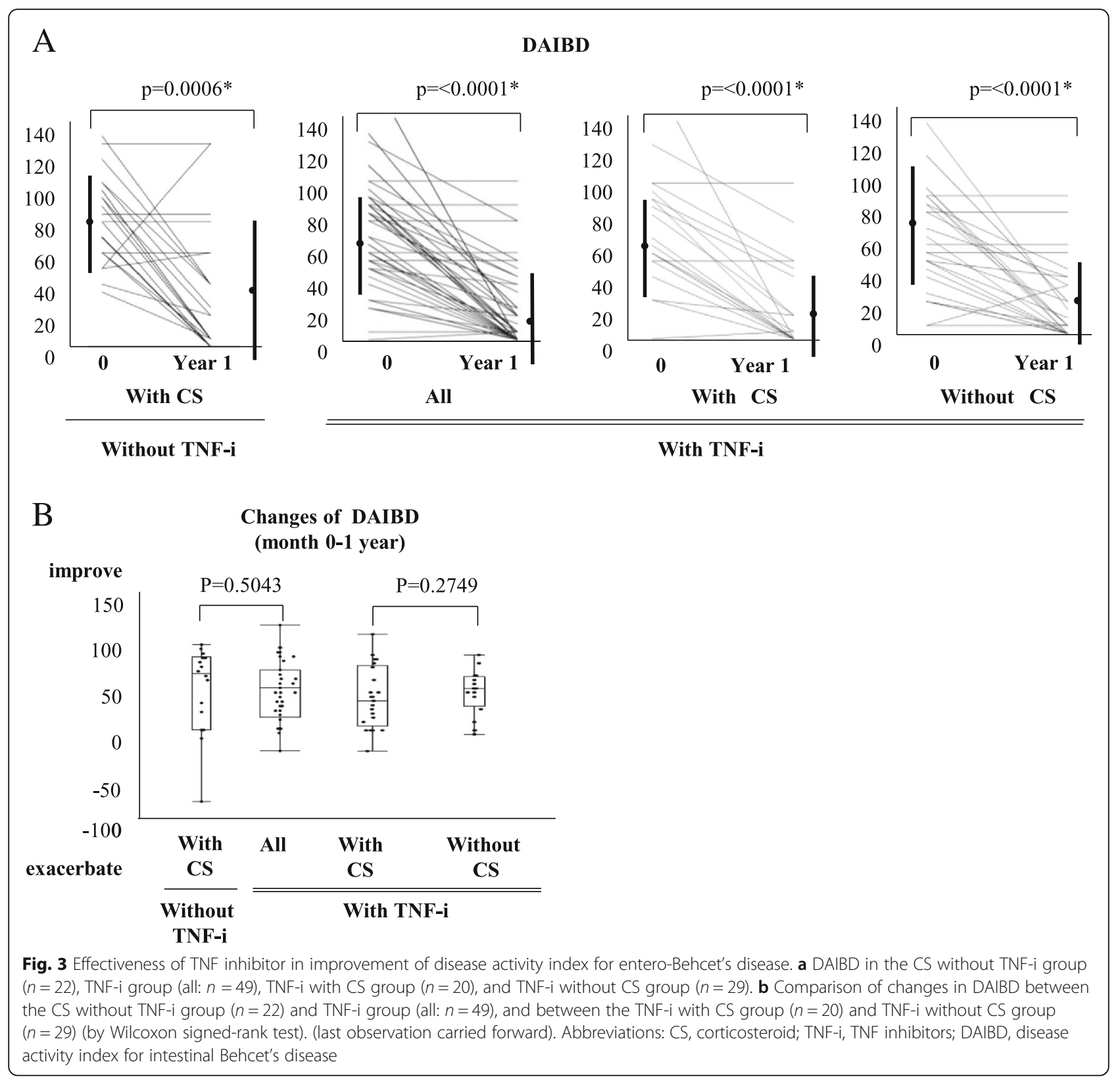

TNF-i and CS discontinued treatment owing to bacterial infection.

\section{Discussion}

In the present study, we introduced TNF-i in 49 patients with refractory entero-BD and followed these patients for 1 year to assess the effectiveness of TNF-i. We also compared the effectiveness of TNF-i to that of CS without TNF-i. When the CS without TNF-i group and the TNF-i group were compared for effectiveness, the ulcer cure and improvement rates, as evaluated by lower gastrointestinal endoscopy, were also significantly lower in the CS without TNF-i group than in the TNF-i group
(Fig. 2a, b). On the other hand, the DAIBD significantly decreased in both groups, with no statistically significant difference. As shown in Fig. 4c, the CS dose, which is considered a risk factor for gastrointestinal bleeding and perforation [16-18], was reduced to $7.5 \mathrm{mg}$ or less in the majority of patients in the TNF-i group; however, in many patients in the CS without TNF-i group, dose reduction of concomitant CS was difficult despite a significant decrease in the DAIBD. In other words, patients in the CS without TNF-i group exhibited CS dependency.

At baseline, the CS without TNF-i group showed significantly higher DAIBD and CS dose than the TNF-i group. Regarding the differences at the baseline, we 


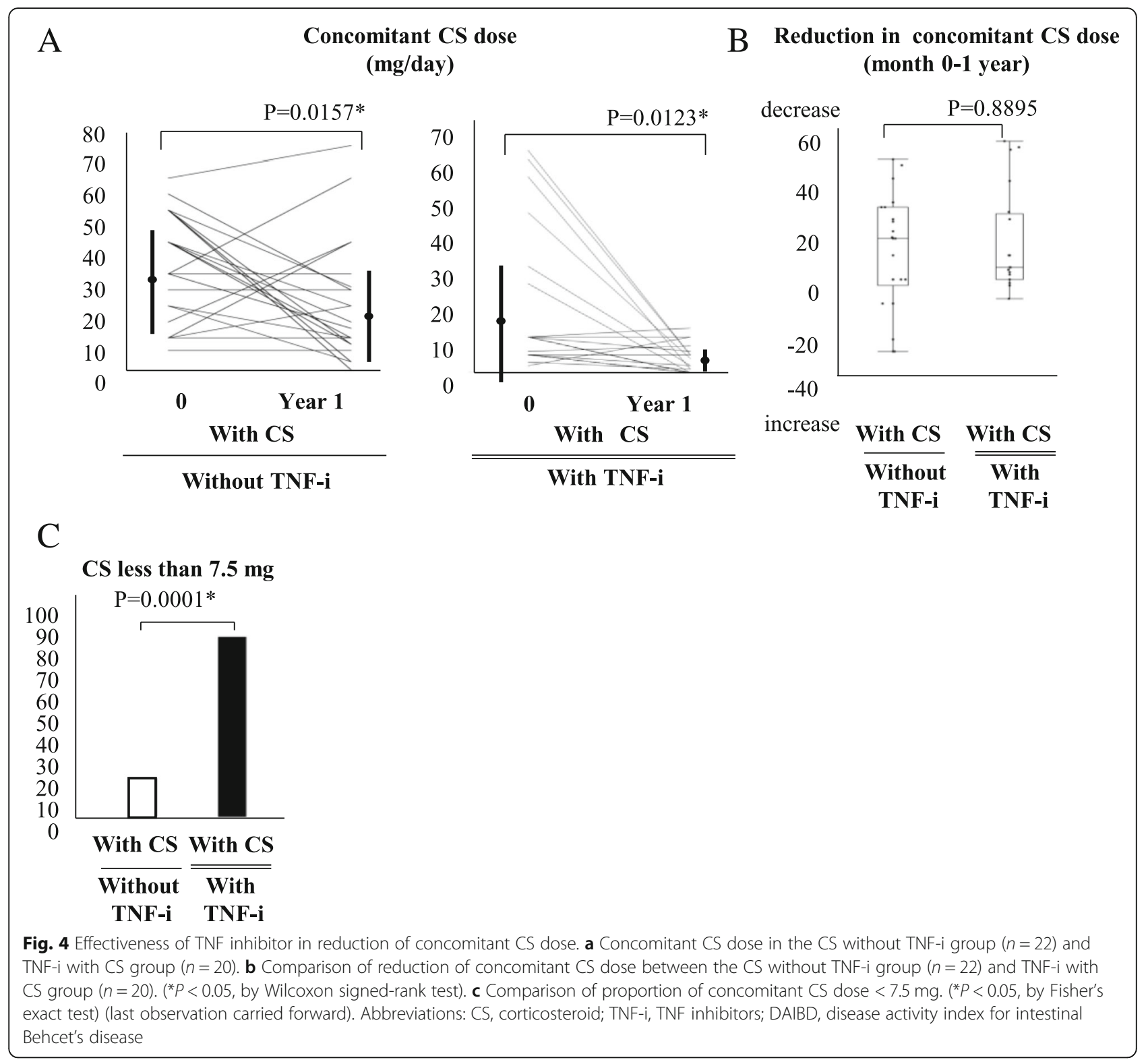

considered that past or recent treatment affected. Some patients had been treated with CS for other symptoms (e.g., skin, mucosa, eyes, genitourinary organs, joints) except entero-BD; some of the patients were under treatment with CS or under dose reduction of CS for entero$\mathrm{BD}$. For these patients, although we newly introduced TNF-i, choices regarding use and dose of CS were made at the physicians' discretion. We considered that as one of the reasons of the difference at the baseline concomitant CS. On the other hand, although no evident predictive factor achieving CS less than $7.5 \mathrm{mg} /$ day was found in our study (Tables 4 and 5), the multivariate analysis revealed that the TNF-i was an independent predictive factor for cure of the intestinal ulcerative lesions (Tables 2 and 3). Comparison of the groups clearly indicated that the use of TNF-i is important not only for reducing disease activity but also for achieving cure of ulcerative lesions.

The TNF-i group was further classified into two groups according to the presence or absence of concomitant CS use (TNF-i with CS group and TNF-i without CS group) to compare the effectiveness of TNF-i. The TNF-i formulations and the necessity of concomitant CS use were, in principle, determined at the discretion of the treating physicians. However, no significant differences in background factors were observed between the two groups at the time of TNF-i introduction (Table 1). Under these study conditions, no statistically significant differences 
Table 4 Comparison of baseline characteristics between the CS less than $7.5 \mathrm{mg} /$ day group and not

\begin{tabular}{|c|c|c|c|}
\hline Variables & $\mathrm{CS} \leqq 7.5 \mathrm{mg}$ at year $1(n=21)$ & Not $(n=21)$ & ${ }^{*} P$ value \\
\hline Male, $n(\%)$ & $6 / 21(28.6 \%)$ & 9/21 (42.9) & 0.5204 \\
\hline Age (years) & $42.8 \pm 13.6$ & $33.1 \pm 10.5$ & $0.0166^{*}$ \\
\hline Disease duration (entero-BD, months) & $42.4 \pm 68.8$ & $40.3 \pm 26.2$ & 0.1341 \\
\hline Multiple ulceration, $n(\%)$ & $4 / 21(19.1 \%)$ & 10/21 (47.6\%) & 0.1001 \\
\hline History of relapse, $n$ (\%) & $11 / 21(52.4 \%)$ & 13/21 (61.9\%) & 0.7557 \\
\hline History of perforation, $n(\%)$ & $3 / 21(14.3 \%)$ & $3 / 21(14.3 \%)$ & 1.0000 \\
\hline History of surgery, n (\%) & $4 / 21(19.1 \%)$ & $3 / 21(14.3 \%)$ & 1.0000 \\
\hline Concomitant CS dose (mg/day) & $22.7 \pm 21.5$ & $28.9 \pm 17.1$ & 0.1450 \\
\hline Concomitant colchicine use, $n(\%)$ & 15/21 (71.4\%) & 10/21 (47.6\%) & 0.2082 \\
\hline Concomitant drug use, $n$ (\%) (MTX, MS/SSZ, AZ) & $18 / 21(85.7 \%)$ & 15/21 (71.4\%) & 0.4537 \\
\hline Concomitant MTX use, $n(\%)$ & 15/21 (71.4\%) & $3 / 21(14.3 \%)$ & $0.0004^{*}$ \\
\hline TNF-i use, $n(\%)$ & 17/21 (81.0\%) & $3 / 21(14.3 \%)$ & $<0.0001^{*}$ \\
\hline DAIBD & $70.0 \pm 40.5$ & $86.7 \pm 25.3$ & 0.0570 \\
\hline
\end{tabular}

Plus-minus values are means \pm standard deviation. ${ }^{*} P<0.05$ by Fisher's exact test or Mann-Whitney $U$ test. $B D$ Behcet's disease, $C S$ corticosteroid, col colchicine, MS mesalazine, SSZ sulfasalazine, MTX methotrexate, AZ azathioprine, TNF- $i$ TNF inhibitors, DAIBD disease activity index for intestinal Behcet's disease

between the TNF-i with CS group and the TNF-i without CS group were observed in any of the following endpoints: ulcer cure rate, ulcer improvement rate, ulcer exacerbation rate, retention rate of TNF-i, disease activity based on DAIBD, and decrease in DAIBD. Although none of the endpoints showed statistically significant differences, the respective rates or scores were also lower in the TNF-i without CS group than in the TNF-i with CS group. Furthermore, as shown in Fig. 1, the TNF-i with CS group included one non-responder to TNF-i, whereas the TNF-i without CS group included three. Although no patient in the TNF-i with CS group experienced relapse after introduction of TNF-i, four patients in the TNF-i without CS group did. No statistically significant differences were observed in the relapse rates or the proportions of nonresponders (TNF-i with CS group 5.0\%, TNF-i without CS group $13.8 \%, P=0.3114$, by Fisher's exact test). Based on these results, although the activity of entero-BD was sufficiently controlled and maintained at low levels with TNF-i therapy without CS in some patients, there may be other patients for whom concomitant CS use is necessary. In addition, the present study failed to identify any

Table 5 Baseline predictive factors of CS less than $7.5 \mathrm{mg} /$ day analyzed by multivariate analysis

\begin{tabular}{|c|c|c|c|c|c|c|}
\hline \multirow[t]{2}{*}{ Variables } & \multicolumn{3}{|c|}{ Univariate logistic regression } & \multicolumn{3}{|c|}{ Multiple logistic regression } \\
\hline & Wald & $P$ & OR $(95 \% \mathrm{Cl})$ & Wald & $P$ & OR $(95 \% \mathrm{Cl})$ \\
\hline Male, $n(\%)$ & 0.9232 & 0.3365 & $0.53(0.15-1.92)$ & & & \\
\hline Age (years) & 5.1680 & 0.0230 & $1.07(1.01-1.14)$ & 2.32 & 0.1135 & $1.06(0.98-1.13)$ \\
\hline Disease duration (entero-BD, months) & 0.0186 & 0.8914 & $1.00(0.99-1.01)$ & & & \\
\hline Multiple ulceration, $n$ (\%) & 3.6556 & 0.1559 & $0.26(0.06-1.03)$ & & & \\
\hline History of relapse, $n(\%)$ & 0.3876 & 0.5336 & $0.53(0.43-5.05)$ & & & \\
\hline History of perforation, $n(\%)$ & 0 & 1.0000 & $1.00(0.18-5.63)$ & & & \\
\hline History of surgery, n (\%) & 0.1704 & 0.6797 & $0.71(0.14-3.64)$ & & & \\
\hline Concomitant CS dose (mg/day) & 1.0678 & 0.3014 & $0.98(0.95-1.02)$ & & & \\
\hline Concomitant colchicine use, $n(\%)$ & 2.4121 & 0.1139 & $2.75(0.767-9.86)$ & & & \\
\hline Concomitant drugs use, $n$ (\%) (MTX, MS/SSZ, AZ) & 1.2318 & 0.2556 & $2.40(0.51-11.3)$ & & & \\
\hline Concomitant MTX use, $n(\%)$ & 11.811 & 0.0006 & $15.0(3.20-70.4)$ & 2.32 & 0.5228 & $2.38(0.16-34.2)$ \\
\hline TNF-i use, $n(\%)$ & 15.033 & $<0.0001$ & $25.5(4.96-131.1)$ & 3.11 & 0.0778 & $10.8(0.77-153.0)$ \\
\hline DAIBD & 2.3752 & 0.1233 & $0.98(0.97-1.00)$ & & & \\
\hline
\end{tabular}

Model $X^{2}$ test $P<0.0001$. BD Behcet's disease, CS corticosteroid, col colchicine, MS mesalazine, SSZ sulfasalazine, MTX methotrexate, $A Z$ azathioprine, TNF- $i$ TNF inhibitors, DAIBD disease activity index for intestinal Behcet's disease 
Table 6 Comparison of baseline characteristics between TNF-i with MTX $(n=40)$ and TNF-i without MTX $(n=9)$

\begin{tabular}{llll}
\hline Variables & TNF-i without MTX $(n=9)$ & TNF-i with MTX $(n=40)$ & $* P$ value \\
\hline Male, $n$ (\%) & $1(11.1 \%)$ & $11(27.5 \%)$ & 0.4203 \\
Age (years) & $43.7 \pm 16.0$ & $40.8 \pm 13.7$ & 0.2659 \\
Disease duration (entero-BD, months) & $54.0 \pm 54.7$ & $52.1 \pm 63.5$ & 0.5520 \\
Multiple ulceration, $n(\%)$ & $5 / 9(55.6 \%)$ & $27 / 40(67.5 \%)$ & 0.7001 \\
History of relapse, $n(\%)$ & $7 / 9(77.8 \%)$ & $22 / 40(55.8 \%)$ & 0.2771 \\
History of perforation, $n(\%)$ & $0 / 9(0 \%)$ & $9 / 40(22.5 \%)$ & 0.1795 \\
History of surgery, $n(\%)$ & $0 / 9(0 \%)$ & $6 / 40(15.0 \%)$ & 0.5765 \\
Concomitant CS use, $n(\%)$ & $6 / 9(66.7 \%)$ & $23 / 40(57.5 \%)$ & 0.7199 \\
Concomitant CS dose (mg/day) & $2.22 \pm 3.63$ & $8.83 \pm 17.2$ & 0.4414 \\
DAIBD at baseline & $61.1 \pm 23.3$ & $65.5 \pm 37.3$ & 0.8361
\end{tabular}

Plus-minus values are means \pm standard deviation. ${ }^{*}<<0.05$ by Fisher's exact test or Mann-Whitney $U$ test. $B D$ Behcet's disease, $C S$ corticosteroid, col colchicine, $M S$ mesalazine, SSZ sulfasalazine, MTX methotrexate, AZ azathioprine, TNF-i TNF inhibitors, DAIBD disease activity index for intestinal Behcet's disease

background factors that could predict which patients would be non-responders or experience relapse in the TNF-i without CS group. Furthermore, we did not find any additive effect of MTX on TNF-i (Tables 6 and 7). In the future, additional studies with a larger sample size may be needed to reveal the features of patients who do or do not require a combination of TNF-i and CS. As shown in Fig. 3, concomitant CS doses were significantly reduced in the TNF-i with CS group. Moreover, as described above, the doses were reduced to $7.5 \mathrm{mg}$ or lower in most patients in the TNF-i with CS group, unlike in the CS without TNF-i CS group. During the observation period, neither serious gastrointestinal bleeding nor perforation occurred in any patients in the TNF-i group. In contrast, one patient in the TNF-i with CS group developed bacterial infection resulting in discontinuation of treatment (this patient had entero-BD associated with myelodysplastic syndrome involving trisomy 8). In contrast, no patients in the TNF-i without CS group developed infection requiring discontinuation of TNF-i. Thus, although combination therapy with CS and TNF-i necessitates caution to prevent infection, the current study suggested that TNF-i therapy without CS might be beneficial in reducing the risk of infection.
Limitations of the present study include a small sample size (49 patients) and a short retrospective observation period (1 year). In the future, studies with a larger sample size and a longer observation period are warranted to assess the long-term efficacy of TNF-i in controlling disease activity and to identify predictive factors to detect patients who do not require concomitant usage of CS.

\section{Conclusions}

In the present study, we administered TNF-i to 49 patients with refractory entero-BD and demonstrated its high efficacy based on both endoscopy results and a quantitative disease activity index. In particular, comparison between the TNF-i group and the CS without TNF-i group revealed that TNF-i is highly effective in curing ulcers and reducing concomitant CS doses and identified as an independent predictive factor for cure of the intestinal ulcerative lesions. Furthermore, TNF-i demonstrated sufficient efficacy in both the TNF-i with CS group and the TNF-i without CS group to control disease activity without concomitant CS in some patients. In contrast, TNF-i therapy with CS was associated with concerns regarding infection, and TNF-i therapy without CS with concerns regarding non-responders and relapse.

Table 7 Comparison of clinical effectiveness between TNF-i with MTX $(n=40)$ and TNF-i without MTX $(n=9)$

\begin{tabular}{llll}
\hline Variables & TNF-i without MTX $(n=9)$ & TNF-i with MTX $(n=40)$ & * $P$ value \\
\hline Cure of ulceration, $n(\%)$ & $4 / 9(44.4 \%)$ & $21 / 40(52.5 \%)$ & 0.7252 \\
Improvement of ulceration, $n(\%)$ & $4 / 9(44.4 \%)$ & $23 / 40(57.5 \%)$ & 0.7126 \\
Exacerbation of ulceration, $n(\%)$ & $2 / 9(22.2 \%)$ & $33 / 40(25 \%)$ & 1.0000 \\
Retention rate of TNF-i, $n(\%)$ & $7 / 9(77.8 \%)$ & $44.5 \pm 37.8$ & 0.6631 \\
Changes of DAIBD (month 0-1 year) & $39.4 \pm 35.7$ & $6.13 \pm 17.4$ & 0.8460 \\
Reduction in concomitant CS dose (month 0-1 year) & $1.67 \pm 3.53$ & $36 / 40(90.0 \%)$ & 0.9191 \\
CS less than 7.5 mg at year 1, $n(\%)$ & $9 / 9(100 \%)$ & 1.0000 \\
\hline
\end{tabular}

Plus-minus values are means \pm standard deviation. ${ }^{*} P<0.05$ by Fisher's exact test or Mann-Whitney $U$ test. $B D$ Behcet's disease, $C S$ corticosteroid, col colchicine, MS mesalazine, SSZ sulfasalazine, MTX methotrexate, AZ azathioprine, TNF-i TNF inhibitors, DAIBD disease activity index for intestinal Behcet's disease 


\section{Abbreviations}

BD: Behcet's disease; CS: Corticosteroid; DAIBD: Disease activity index for intestinal Behcet's disease; entero-BD: Entero-Behcet's disease; HLA: Human leukocyte antigen; TNF: Tumor necrosis factor; TNF-i: TNF inhibitor

\section{Acknowledgements}

The authors thank the study participants, without whom this study would never have been accomplished, as well as the investigators for their participation in the study, especially those in Kitakyushu General Hospital Tobata General Hospital, Saiseikai Shimonoseki General Hospital, Fukuoka Yutaka Central Hospital, Nakama Municipal Hospital, and Steel Memorial Yawata Hospital.

\section{Authors' contributions}

IM contributed to the study design, overall review, and writing of the manuscript, and the other authors were involved in the performance of the study and review of the manuscript. YT, KN, SI, SN, and KS participated in the study design and coordination. All authors read and approved the final manuscript.

\section{Funding}

This work was supported in part by Research on rare and intractable diseases and Research Grant-In-Aid for Scientific Research by the Ministry of Health, Labor and Welfare of Japan, the Ministry of Education, Culture, Sports, Science and Technology of Japan, the Japan Agency for Medical Research and Development, the University of Occupational and Environmental Health, Japan, and University of Occupational and Environmental Health Grant for Advanced Research.

\section{Availability of data and materials}

Not applicable.

\section{Ethics approval and consent to participate}

Ethical approval was obtained from the University of Occupational and Environmental Health, Japan Ethics Committee following the Helsinki Declaration. This retrospective study was approved by the institutional review board, and the requirement to obtain informed consent was waived.

\section{Consent for publication}

Not applicable.

\section{Competing interests}

Y. Tanaka has received speaking fees and/or honoraria from Daiichi-Sankyo, Astellas, Eli Lilly, Chugai, Sanofi, Abbvie, Pfizer, YL Biologics, Bristol-Myers, Glaxo-Smithkline, UCB, Mitsubishi-Tanabe, Novartis, Eisai, Takeda, Janssen, and Asahi-kasei and has received research grants from Mitsubishi-Tanabe, Bristol-Myers, Eisai, Chugai, Takeda, Abbvie, Astellas, Daiichi-Sankyo, Ono, MSD, and Taisho-Toyama.

K. Nakano has received speaking fees from Astellas, UCB, Mitsubishi-Tanabe, and Eisai and has received research grants from Mitsubishi-Tanabe, Eisai, and Eli Lilly.

S. Nakayamada received speaking fees and/or honoraria from Bristol-Myers, Sanofi, Abbvie, Eisai, Eli Lilly, Chugai, Asahi-kasei, and Pfizer (less than \$10,000 each) and also research grants from Mitsubishi-Tanabe, Takeda, Novartis, and MSD.

Received: 25 January 2019 Accepted: 6 June 2019

Published online: 22 June 2019

\section{References}

1. O'Duffy JD. Behcet's disease. Curr Opin Rheumatol. 1994;6:39-43.

2. Iwata S, Saito K, Yamaoka K, Tsujimura S, Nawata M, Hanami K, et al. Efficacy of combination therapy of anti-TNF-a antibody infliximab and methotrexate in refractory entero- Behcet's disease. Mod Rheumatol. 2011;21:184-91.

3. Iwata S, Saito K, Yamaoka K, Tsujimura S, Nawata M, Suzuki K, et al. Effects of anti-TNF-alpha antibody infliximab in refractory entero- Behcet's disease. Rheumatology. 2009;49:1012-3

4. Inoue N, Kobayashi K, Naganuma M, Hirai F, Ozawa M, Arikan D, et al. Long-term safety and efficacy of adalimumab for intestinal Behçet's disease in the open label study following a phase 3 clinical trial. Intest Res. 2017;15:395-401.
5. Hibi T, Hirohata S, Kikuchi H, Tateishi U, Sato N, Ozaki K, et al. Infliximab therapy for intestinal, neurological, and vascular involvement in Behcet disease: efficacy, safety, and pharmacokinetics in a multicenter, prospective, open-label, single-arm phase 3 study. Medicine (Baltimore). 2016;95:e3863.

6. Tanida S, Inoue N, Kobayashi K, Naganuma M, Hirai F, lizuka B, et al. Adalimumab for the treatment of Japanese patients with intestinal Behçet's disease. Clin Gastroenterol Hepatol. 2015;13:940-8.

7. Lee JH, Cheon JH, Jeon SW, Ye BD, Yang SK, Kim YH, et al. Efficacy of infliximab in intestinal Behçet's disease: a Korean multicenter retrospective study. Inflamm Bowel Dis. 2013;19:1833-8.

8. Vallet H, Riviere S, Sanna A, Deroux A, Moulis G, Addimanda O, et al. Efficacy of anti-TNF alpha in severe and/or refractory Behçet's disease: multicenter study of 124 patients. J Autoimmun. 2015;62:67-74.

9. Hisamatsu T, Ueno F, Matsumoto T, Kobayashi K, Koganei K, Kunisaki R, et al. The 2nd edition of consensus statements for the diagnosis and management of intestinal Behçet's disease: indication of anti-TNFa monoclonal antibodies. J Gastroenterol. 2014;49:156-62.

10. Lee HW, Kim WH, Cheon JH. The medical treatments of intestinal Behcet's disease: an update. Intest Res. 2013;11:155-60.

11. Park YE, Cheon JH. Updated treatment strategies for intestinal Behçet's disease. Korean J Intern Med. 2018;33:1-19.

12. Cheon JH, Han DS, Park JY, Ye BD, Jung SA, Park YS ea. Development, validation, and respomsiveness of a novel disease activity index for intestinal Behcet's disease. Inflamm Bowel Dis. 2011;17:605-13.

13. Lee HJ, Kim YN, Jang HW, Jeon HH, Jung ES, Park SJ, et al. Correlations between endoscopic and clinical disease activity indices in intestinal Behcet's disease. World J Gastroenterol. 2012;28(18):5771-8.

14. Suzuki Kurokawa M, Suzuki N. Behcet's disease. Clin Exp Med. 2004;4:1-20.

15. [No author listed]. Criteria for diagnosis of Behcet's disease. International Study Group for Behcet's Disease. Lancet 1990;335:1078-1080.

16. Jung YS, Yoon JY, Lee JH, Jeon SM, Hong SP, Kim TI, et al. Prognostic factors and long-term clinical outcomes for surgical patients with intestinal Behcet's disease. Inflamm Bowel Dis. 2011:17:1594-602.

17. Narum S, Westergren T, Klemp M. Corticosteroids and risk of gastrointestinal bleeding: a systematic review and meta-analysis. BMJ Open. 2014:4:e004587.

18. Park J, Cheon JH, Park YE, Lee YJ, Lee HJ, Park SJ, et al. Risk factors and outcomes of acute lower gastrointestinal bleeding in intestinal Behcet's disease. Int J Color Dis. 2017;32:745-51.

\section{Publisher's Note}

Springer Nature remains neutral with regard to jurisdictional claims in published maps and institutional affiliations.

Ready to submit your research? Choose BMC and benefit from:

- fast, convenient online submission

- thorough peer review by experienced researchers in your field

- rapid publication on acceptance

- support for research data, including large and complex data types

- gold Open Access which fosters wider collaboration and increased citations

- maximum visibility for your research: over $100 \mathrm{M}$ website views per year

At $\mathrm{BMC}$, research is always in progress.

Learn more biomedcentral.com/submissions 